\title{
Processes with the $t$-channel singularity in the physical region: finite beam sizes make cross sections finite
}

\author{
K. Melnikov \\ Institut für Physik,Universität Mainz* \\ and \\ V.G. Serbo \\ Institut für Theoretische Physik, Universität Leipzig ${ }^{\dagger}$
}

\begin{abstract}
It is known that some high-energy processes have a $t$-channel singularity in the physical region. In this paper we show that this singularity is regularized if one takes into account the finite sizes of the colliding beams, i.e. the realistic situation which takes place at high-energy colliders. On this way we obtain the finite cross section which is linear proportional to the transverse sizes of the colliding beams. As an application of the above result we study the production of the single $W$ boson at $\mu^{+} \mu^{-}$colliders in the reaction $\mu^{-} \mu^{+} \rightarrow e \bar{\nu}_{e} W^{+}$. For the total energy $\sqrt{s} \approx 95$ $\mathrm{GeV}$ and reasonable parameters of the muon beams the "beam-size" dependent part of the cross section for this reaction is of the order of the standard cross section of the $\mu^{-} \mu^{+} \rightarrow \mu^{-} \bar{\nu}_{\mu} W^{+}$process.
\end{abstract}

MZ-TH/96-03

${ }^{*}$ D 55099 Germany, Mainz, Johannes Gutenberg Universität, Institut für Physik, THEP, Staudinger weg 7; e-mail: melnikov@dipmza.physik.uni-mainz.de

†Permanent address: Novosibirsk State University, 630090, Novosibirsk, Russia; e-mail: serbo@phys.nsu.nsk.su 


\section{Introduction}

It is known since early 60's that some high-energy processes can have a $t$-channel singularity in the physical region [1]. This situation can occur if initial particles in a given reaction are unstable and the masses of the final particles are such that the real decay of the initial particles can take place.

Recently in the paper [2] it was stressed that this problem turned out to be a practical issue for the reaction $\mu^{-} \mu^{+} \rightarrow e^{-} \bar{\nu}_{e} W^{+}$. It is noted in [2] that the standard calculation of this cross section leads to the infinite result. Indeed, if the invariant mass of the final $e \bar{\nu}_{e}$ system is smaller than the muon mass $m$, the square of the momentum transfer in the $t$-channel $q^{2}$ can be both positive and negative depending on the scattering angles. In the region of small $q^{2}$ defined by the inequalities $-\Lambda<q^{2}<\Lambda$ and $\Lambda \ll m^{2}$ the main contribution to the discussed cross section is given by the diagram with the exchange of the muonic neutrino in the $t$-channel (see fig.1). The $t$-channel propagator brings the factor $1 / q^{2}$ to the matrix element $M$ of this process

$$
M \propto \frac{1}{q^{2}+i \epsilon}
$$

which results in the power-like singularity in the standard cross section

$$
d \sigma \propto \int_{-\Lambda}^{\Lambda}|M|^{2} d q^{2} \propto B=\int_{-\Lambda}^{\Lambda} \frac{d q^{2}}{\left|q^{2}+i \epsilon\right|^{2}}=\infty
$$

It is, therefore, necessary to find physically reliable method to produce definite prediction for the measurable number of events.

In the paper [2] (see also [1]) it was suggested to regularize this divergence by taking into account the instability of muons in the initial state. If this is the case, then the muon mass is the complex quantity with the imaginary part proportional to the muon width $\Gamma$. It is possible then to solve the energy-momentum conservation constraints with the conclusion that the square of the momentum transfer in the $t$-channel acquires imaginary part proportional to the muon width. This observation leads to the replacement

$$
q^{2} \rightarrow q^{2}-i \gamma, \quad \gamma \sim m \Gamma
$$

The divergent integral in (2) is therefore regularized

$$
B=\int_{-\Lambda}^{\Lambda} \frac{d q^{2}}{\left|q^{2}+i \epsilon\right|^{2}} \rightarrow \int_{-\Lambda}^{\Lambda} \frac{d q^{2}}{\left|q^{2}-i \gamma\right|^{2}} \sim \frac{1}{\gamma} \sim \frac{1}{m \Gamma}
$$

Consequently direct calculation of the scattering cross section becomes possible.

We note in this respect that if such a regularization is performed, it is possible to estimate the typical time necessary for this reaction to occur. It is not difficult to see that this time is of the order of the moving muon life time, i.e. much more larger than the time necessary for muon beams to cross each other. Therefore for the realistic muon colliders the results of ref. 22] are not applicable and a new consideration is necessary.

We have found that for the realistic muon colliders there exist much more important effect connected with the finite beam sizes. In this paper we are going to show that 
accounting for the finite sizes of the colliding beams gives a finite cross sections for the processes like

$$
\mu^{-} \mu^{+} \rightarrow e^{-} \bar{\nu}_{e} X
$$

with the t-channel singularity in the physical region. This is the main result of our paper. As an example, we reconsider reaction $\mu^{-} \mu^{+} \rightarrow e \bar{\nu}_{e} W^{+}$and show that the actual cross section of this process for the total energy $\sqrt{s} \sim 100 \mathrm{GeV}$ is approximately $1 \mathrm{fb}$ for the typical transverse beam sizes $a \sim 10^{-3} \mathrm{~cm}$.

Let us mention here that the effect of the finite beam size at the high-energy colliders is well studied both experimentally and theoretically (for the review see ref. [3]). For the first time this beam-size effect (BSE) was observed at the VEPP-4 collider (Novosibirsk) in 1980-81 during the study of the single bremsstrahlung in the electron-positron collisions [4]. This year the BSE was observed at HERA in the reaction $e p \rightarrow e p \gamma$ [5]. In both cases the number of observed photons was smaller than it was expected according to the standard calculations. The decreased number of photons was explained by the fact that impact parameters, which gave essential contribution to the standard cross section of these reactions, were larger by 2-3 orders of magnitude as compared to the transverse beam sizes. From the theoretical point of view, the BSE represents a remarkable example of the situation where traditional notion of the cross section and the standard formulae for the number of events are not valid.

Let us briefly outline the principal points of our consideration. The standard rules for calculations in high-energy physics correspond to the collision of plane waves which represent initial states of colliding particles. Instead of this we consider collision of the wave packets which correspond to the particle beams in the initial state. As a consequence the standard rules are modified (see detailed discussion in the section 3). For instance, in the eq.(2) the following replacement takes place:

$$
|M|^{2} \rightarrow M_{f i} M_{f i^{\prime}}^{*}
$$

Here $M_{f i}$ and $M_{f i^{\prime}}$ are the matrix elements with identical final but different initial states. The difference in the momenta of the initial states is of the order of $1 / a$ for the beam of the transverse size $a$. Due to this fact the singular propagators $1 / q^{2}$ (which are the reason for the pathological behaviour of the cross section) are now taken in the different points of the momentum space for the amplitudes $M_{f i}$ and $M_{f i^{\prime}}$ :

$$
M_{f i} \propto \frac{1}{q^{2}-\lambda+i \epsilon}, \quad M_{f i^{\prime}} \propto \frac{1}{q^{2}+\lambda+i \epsilon}, \quad \lambda \sim \frac{m}{a} .
$$

Consequently the infinite factor $B$ is replaced by:

$$
B=\int_{-\Lambda}^{\Lambda} \frac{d q^{2}}{\left|q^{2}+i \epsilon\right|^{2}} \rightarrow \sim \int_{-\Lambda}^{\Lambda} \frac{d q^{2}}{\left(q^{2}-\lambda+i \epsilon\right)\left(q^{2}+\lambda-i \epsilon\right)}
$$

Below we show that

$$
B \sim \frac{1}{\lambda} \sim \frac{a}{m}
$$

and that the main contribution to this integral is given by the pole of the neutrino propagator $q^{2}+\lambda-i \epsilon=0$, i.e. by the real neutrino. This means [6] that the effect under discussion is connected with the decay $\mu^{-} \rightarrow e^{-} \bar{\nu}_{e} \nu_{\mu}$ and subsequent scattering of the real 
muonic neutrinos on the $\mu^{+}$beam. As discussed in detail below these neutrinos have a broad energy spectrum (see fig. 2)

$$
d N_{\nu}=N_{\nu} f(x) d x
$$

( $x$ is the fraction of the $\mu^{-}$energy carried by neutrino). In accordance with the eq.(17), the total number of these neutrinos (per one muon) is proportional to the typical transverse beam size $a$ :

$$
N_{\nu} \sim \frac{a}{c \tau}
$$

where $\tau=1 / \Gamma$ is the muon life time, $c \tau=660 \mathrm{~m}$. As a consequence the "non-standard" contribution to the cross section is also proportional to the transverse beam size

$$
\sigma_{\text {non-stand }}=N_{\nu} \int f(x) \sigma_{\nu \mu \rightarrow W}(x s) d x \propto a .
$$

Let us mention here that in all the previous cases when the effects of the finite beam sizes have been studied, the results depend logarithmically on the beam size:

$$
\sigma \propto \ln a .
$$

In contrast to this, in our case ( $t$-channel singularity in the physical region ) the cross section (10) is linear proportional to the transverse sizes of the colliding beams.

The subsequent part of the paper is organized as follows: in the next section, as a realistic example of the processes with the $t$-channel singularity in the physical region, we consider reaction (4) and present its cross section; in the section 3 we prove the basic formula used in the section 2 ; section 4 is devoted to the particular case - the reaction $\mu^{-} \mu^{+} \rightarrow e \bar{\nu}_{e} W^{+}$; in the section 5 we present our conclusions; Appendix is devoted to the detailed consideration of a model process with a $t$-channel singularity in the physical region.

\section{General case}

In this section we show how the cross section of the reaction $\mu^{-} \mu^{+} \rightarrow e^{-} \bar{\nu}_{e} X$ can be calculated. We introduce the following notations: $s=\left(p_{1}+p_{2}\right)^{2}=4 E^{2}$ is the square of the total energy in the center of mass frame, $m$ and $\Gamma=1 / \tau$ are muon mass and width respectively, $p_{1}^{2}=p_{2}^{2}=m^{2}, p_{3}$ is the 4-momentum of the final $e^{-} \bar{\nu}_{e}$ system, $y=p_{3}^{2} / m^{2}$, $q=p_{1}-p_{3}=(\omega, \mathbf{q})$ is the momentum transfer in the $t$-channel and $x=q p_{2} / p_{1} p_{2} \approx \omega / E$.

From simple kinematics it follows that

$$
q^{2}=-\frac{\mathbf{q}_{\perp}^{2}}{1-x}+t_{0}, \quad t_{0}=\frac{x(1-x-y)}{1-x} m^{2}
$$

where $\mathbf{q}_{\perp}$ is the component of the momentum $\mathbf{q}$ which is transverse to the momenta of the initial muons. Note, that

$$
t_{0}>0 \text { for } y<1-x
$$

and that for $q^{2}=0$

$$
\left|\mathbf{q}_{\perp}\right|=q_{\perp}^{0}=m \sqrt{x(1-x-y)} .
$$


For $y<1-x\left(\right.$ or $\left.t_{0}>0\right)$ we write the cross section in the form

$$
d \sigma=d \sigma_{\text {stand }}+d \sigma_{\text {non-stand }}
$$

where by definition $d \sigma_{\text {stand }}$ corresponds to the region $q^{2}<-m^{2}$ and $d \sigma_{\text {non-stand }}$ corresponds to the region

$$
-m^{2}<q^{2}<t_{0}
$$

We will show that the main contribution to $d \sigma_{\text {non-stand }}$ is given by the region of a very small values of $q^{2}$ inside the region (16):

$$
-\Lambda<q^{2}<\Lambda, \quad m / a \ll \Lambda \ll m^{2}
$$

where $a$ is the typical transverse beam size. In the region (17) the main contribution comes from the diagram with the exchange of the muonic neutrino in the $t$-channel (fig.1). Since for such $q^{2}$ the exchanged neutrino is almost real, the corresponding matrix element can be considerably simplified.

First, the numerator of the neutrino propagator $\hat{q}$ can be presented in the form

$$
\hat{q} \approx \sum_{\lambda} u_{\lambda}(\mathbf{q}) \bar{u}_{\lambda}(\mathbf{q})
$$

Here $u_{\lambda}(\mathbf{q})$ is the Dirac spinor for the massless fermion with momentum $\mathbf{q}$ and helicity $\lambda$. Second, in the region of interest the upper block of the diagram (see fig.1) corresponds to the decay $\mu^{-} \rightarrow e \bar{\nu}_{e} \nu_{\mu}$. In this decay only left-handed $\nu_{\mu}$ is produced. Therefore in the above sum the only term with $\lambda=-1 / 2$ is relevant.

As a result, in the region (17) we present the matrix element $M$ in the form

$$
M=-M_{\mu \rightarrow e \bar{\nu}_{e} \nu_{\mu}} \frac{1}{q^{2}+i \epsilon} M_{\nu \mu \rightarrow X}
$$

where $M_{\mu \rightarrow e \bar{\nu}_{e} \nu_{\mu}}$ is the matrix element for the $\mu^{-}$decay and $M_{\nu \mu \rightarrow X}$ is the matrix element for the $\nu_{\mu} \mu^{+} \rightarrow X$ process. In both of these subprocesses we take $q^{2}$ equal to zero.

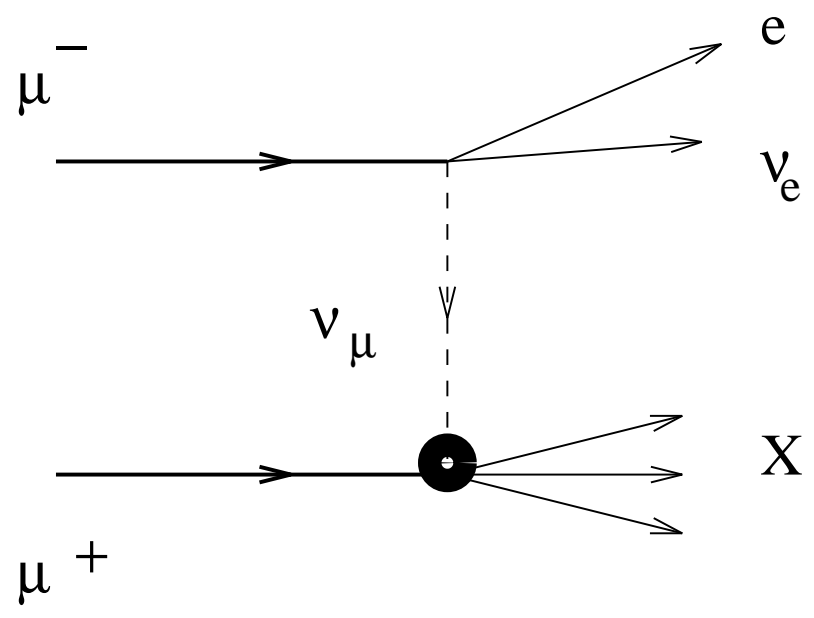

Figure 1: The Feynman diagram for the reaction $\mu^{-} \mu^{+} \rightarrow e \bar{\nu}_{e} X$ which gives the leading contribution in the region of small $\left|q^{2}\right|$. 
Using this equation we express the non-standard contribution through the muon decay width $\Gamma$ and the cross section $\sigma_{\nu \mu \rightarrow X}$ of the $\nu_{\mu} \mu^{+} \rightarrow X$ process as:

$$
d \sigma_{\text {non-stand }}=\frac{1}{\pi} x m d \Gamma \frac{d q^{2}}{\left|q^{2}\right|^{2}} d \sigma_{\nu \mu \rightarrow X} .
$$

For unpolarized muon beams we have

$$
d \Gamma=\frac{\Gamma}{\pi}(1-y)(1+2 y) d x d y d \varphi
$$

where $\varphi$ is the azimuthal angle of the vector $\mathbf{q}$. Let us call the coefficient in front of $d \sigma_{\nu \mu \rightarrow X}$ in eq. (19) as the number of neutrinos:

$$
d N_{\nu}=\frac{1}{\pi} x m d \Gamma \frac{d q^{2}}{\left|q^{2}\right|^{2}}=\frac{m \Gamma}{\pi^{2}} x(1-y)(1+2 y) d x d y d \varphi \frac{d q^{2}}{\left|q^{2}\right|^{2}}
$$

As it is clear from the eq.(19), the standard calculation of the cross section turns out to be impossible due to the power-like singularity, since the point $q^{2}=0$ is within the physical region for $y<1-x$.

The main result of our investigation of the BSE in the above process can be formulated as follows:

Accounting for the BSE results in the following treatment of the divergent integral in the eq.(19):

$$
B=\int_{-\Lambda}^{\Lambda} \frac{d q^{2}}{\left|q^{2}\right|^{2}} \rightarrow \pi \frac{a}{q_{\perp}^{0}}=\pi \frac{a}{m \sqrt{x(1-x-y)}}
$$

The exact expression for the quantity $a$ will be given below (see eqs.(42)-(45)). We just mention here that it is proportional to the transverse sizes of the colliding beams. For the identical round Gaussian beams with the root-mean-square radii

$$
\sigma_{i x}=\sigma_{i y}=\sigma_{\perp}, \quad i=1,2
$$

this quantity is equal to

$$
a=\sqrt{\pi} \sigma_{\perp} .
$$

Hence, the quantity $B \sim a / m$ as it is noted in (7). The contribution (22) comes from the region (17). It is not difficult to estimate that the contribution from the rest part of the region (16) is smaller. Indeed, its relative value is of the order of

$$
\frac{1}{B} \int_{-m^{2}}^{-\Lambda} \frac{d q^{2}}{\left|q^{2}\right|^{2}}+\frac{1}{B} \int_{\Lambda}^{t_{0}} \frac{d q^{2}}{\left|q^{2}\right|^{2}} \sim \frac{m / a}{\Lambda} \ll 1
$$

Using eqs. (22) $-(24)$ and integrating the number of neutrinos (21) over $y$ (in the region $0<y<1-x)$ and over $\varphi$, we arrive to the following spectrum of neutrinos:

$$
\frac{d N_{\nu}(x)}{d x}=\frac{\pi a}{2 c \tau} f(x), \quad f(x)=\frac{24}{5 \pi} \sqrt{x(1-x)}\left(1+\frac{22}{9} x-\frac{16}{9} x^{2}\right)
$$


where $\tau$ is the life time of the muon at rest, $c \tau=660 \mathrm{~m}$. The function $f(x)$ is normalized to 1 :

$$
\int_{0}^{1} f(x) d x=1 .
$$

The plot of this function is presented in the fig.2. The total number of neutrinos is equal to

$$
N_{\nu}=\frac{\pi}{2} \frac{a}{c \tau}
$$

After the spectrum of neutrinos is obtained, the non-standard cross section for the reaction $\mu^{-} \mu^{+} \rightarrow e \bar{\nu}_{e} X$ is given by the equation

$$
d \sigma_{\text {non-stand }}=d N_{\nu}(x) d \sigma_{\nu \mu \rightarrow X}(x s)=N_{\nu} f(x) d x d \sigma_{\nu \mu \rightarrow X}(x s) .
$$

Subsequent integration over $x$ can be performed without further difficulties. For the particular case $X=W^{+}$such calculation is performed in the section 4 .

\section{Derivation of the basic formula}

Here we are going to prove our result presented in the previous section (see eq.(22)).

To begin with, let us note that the standard notion of the cross section is an approximation itself. As is well known, it corresponds to the plane waves approximation for initial and final particles. In the real experiments the particles are confined to the beams of a relatively small size and it is the collision of such beams that leads to the measurable number of events.

In order to arrive to a more general formulae for the scattering processes, it is necessary to describe the collisions of the wave packets instead of the plane waves. In view of the fact that the movement of the particles inside a beam is quasiclassical, simple and efficient technique for taking into account the beam-size effects in the actual calculations has been developed [7] (for the review see [3]).

Below we present some results from the refs. [7], [3] which are essential for our discussion. For simplicity, we neglect the energy and angular spread of the particles in the colliding beams.

Let us remind that in the standard approach the number of events $N$ is the product of the cross section $\sigma$ and the luminosity $L$ :

$$
d N=d \sigma L, \quad d \sigma \propto|M|^{2}, \quad L=v \int n_{1}(\mathbf{r}, t) n_{2}(\mathbf{r}, t) d^{3} r d t
$$

where $v=\left|\mathbf{v}_{1}-\mathbf{v}_{2}\right|=2$ for the head-on collision of the ultra-relativistic beams. The quantities $n_{i}(\mathbf{r}, t)$ are the particle densities of the beams.

The transformation from the plane waves to the wave packets results in the following changes. The squared matrix element $|M|^{2}$ with the initial state in the form of the plane waves with the momenta $\mathbf{p}_{\mathbf{1}}$ and $\mathbf{p}_{\mathbf{2}}$ transforms to the product of the matrix elements $M_{f i}$ and $M_{f i^{\prime}}^{*}$ with the different initial states:

$$
d \sigma \propto|M|^{2} \rightarrow d \sigma(\boldsymbol{\kappa}) \propto M_{f i} M_{f i^{\prime}}^{*}
$$

Here the initial state $|i\rangle$ is the direct product of the plane waves with the momenta $\mathbf{k}_{1}=\mathbf{p}_{1}+\frac{1}{2} \boldsymbol{\kappa}$ and $\mathbf{k}_{2}=\mathbf{p}_{2}-\frac{1}{2} \boldsymbol{\kappa}$, while the initial state $\left|i^{\prime}\right\rangle$ is the direct product of 
the plane waves with the momenta $\mathbf{k}_{1}^{\prime}=\mathbf{p}_{1}-\frac{1}{2} \boldsymbol{\kappa}$ and $\mathbf{k}_{2}^{\prime}=\mathbf{p}_{2}+\frac{1}{2} \boldsymbol{\kappa}$. Instead of the luminosity $L$ the number of events starts to depend on the quantity

$$
L(\varrho)=v \int n_{1}(\mathbf{r}, t) n_{2}(\mathbf{r}+\varrho, t) d^{3} r d t
$$

through the following formula

$$
d N=\int \frac{d^{3} \kappa d^{3} \varrho}{(2 \pi)^{3}} \mathrm{e}^{i \kappa \varrho} d \sigma(\boldsymbol{\kappa}) L(\boldsymbol{\varrho}) .
$$

The characteristic values of $\kappa$ are of the order of the inverse beam sizes, i.e.

$$
\kappa \sim \frac{1}{a}
$$

Usually this quantity is much smaller than the typical scale for the variation of the matrix element with respect to the initial momenta. In this case we can put $\kappa=0$ in $d \sigma(\boldsymbol{\kappa})$ which results in the standard expression for the number of events (28). Otherwise, one should analyze the complete formulae which take into account the effect of the finite beam sizes.

In view of the discussion given in the previous section, this is indeed the situation which occurs in our case. Now we want to show how the finite result for the number of events can be obtained starting from the complete formula (31).

Let us first define the "observable cross section" by the relation (compare with the eq.(15))

$$
d \sigma=\frac{d N}{L}=d \sigma_{\text {stand }}+d \sigma_{\text {non-stand }}
$$

where $L$ is the standard luminosity. By writing the number of events in such a way, we push the BSE to the quantity $d \sigma_{\text {non-stand }}$.

The study of the matrix element of the discussed process (18) suggests that the only quantity sensitive to the small variation of the initial momenta is the denominator of the neutrino propagator for small values of $q^{2}$. Consequently, the transformations (29)-(31) reduces to the following modification:

$$
\frac{1}{\left|q^{2}\right|^{2}} \rightarrow \frac{1}{t+i \epsilon} \frac{1}{t^{\prime}-i \epsilon}
$$

where

$$
q^{2}=\left(p_{1}-p_{3}\right)^{2}, \quad t=\left(k_{1}-p_{3}\right)^{2}, \quad t^{\prime}=\left(k_{1}^{\prime}-p_{3}\right)^{2} .
$$

Let us expand $t$ and $t^{\prime}$ up to the terms linear in $\boldsymbol{\kappa}$. This gives

$$
t=q^{2}-\lambda, \quad t^{\prime}=q^{2}+\lambda
$$

where

$$
\lambda=\kappa \mathbf{Q}, \quad \mathbf{Q}=-\mathbf{p}_{3}+\frac{E_{3}}{E_{1}} \mathbf{p}_{1} .
$$

In the center of mass frame of the colliding muons

$$
\mathbf{Q} \approx-\mathbf{p}_{3 \perp}=\mathbf{q}_{\perp}=\left|\mathbf{q}_{\perp}\right| \mathbf{n}, \quad \mathbf{n}=(\cos \varphi, \sin \varphi, 0) .
$$


As a result, the quantity $B$ in the eq. (22) transforms to (compare with the eq. (6))

$$
B=\int \frac{d^{3} \kappa d^{3} \varrho}{(2 \pi)^{3}} \mathrm{e}^{i \kappa \varrho} \frac{L(\varrho)}{L} \int_{-\Lambda}^{\Lambda} \frac{d q^{2}}{\left(q^{2}-\lambda+i \epsilon\right)\left(q^{2}+\lambda-i \epsilon\right)}
$$

It has already been mentioned that the above equation must be understood in the sense that we isolate the part, which does not possess the limit $\kappa \rightarrow 0$, to the quantity $B$. All other quantities are smooth in the above limit and hence do not present any problem.

To perform the $q^{2}$-integration in (38) we note that in the region (17)

$$
\lambda=\boldsymbol{\kappa} \mathbf{n} \sqrt{\left(q_{\perp}^{0}\right)^{2}-(1-x) q^{2}} \approx \lambda_{0}=\boldsymbol{\kappa} \mathbf{n} q_{\perp}^{0} .
$$

This approximation is justified because

$$
\frac{\left|q^{2}\right|}{m^{2}} \lesssim \frac{\Lambda}{m^{2}} \ll 1
$$

Then we extend the region of integration over $q^{2}$ up to $\pm \infty$. The error introduced by this procedure is of the order of $(m / a) / \Lambda \ll 1$ (cf. eq.(24)). Now the integral over $q^{2}$ along the real axis can be replaced by the integral over the contour $C$ which goes around the upper half plane of the complex variable $q^{2}$ :

$$
\int_{-\infty}^{\infty} \frac{d q^{2}}{D}=\int_{C} \frac{d q^{2}}{D}=\frac{\pi i}{-\lambda_{0}+i \epsilon}
$$

Here

$$
D=\left(q^{2}-\lambda_{0}+i \epsilon\right)\left(q^{2}+\lambda_{0}-i \epsilon\right) .
$$

The result is provided by the pole $q^{2}+\lambda_{0}-i \epsilon=0$ in the upper half plane.

Further integrations are simply performed with the help of the exponential representation

$$
\frac{i}{-\lambda_{0}+i \epsilon}=\int_{0}^{\infty} \mathrm{e}^{i \alpha\left(-\lambda_{0}+i \epsilon\right)} d \alpha
$$

Subsequent integrations over $\boldsymbol{\kappa}$ and $\varrho$ become trivial and we obtain

$$
B=\pi \int_{0}^{\infty} \frac{L\left(\alpha \mathbf{n} q_{\perp}^{0}\right)}{L} d \alpha
$$

After simple redefinition $\alpha q_{\perp}^{0}=\varrho$ we get for the factor $B$ :

$$
B=\pi \frac{a}{q_{\perp}^{0}}, \quad a=\int_{0}^{\infty} \frac{L(\varrho \mathbf{n})}{L} d \varrho, \quad \mathbf{n}=\frac{\mathbf{Q}}{|\mathbf{Q}|} \approx \frac{\mathbf{q}_{\perp}^{0}}{q_{\perp}^{0}}
$$

This completes the proof of the eq. (22).

\footnotetext{
${ }^{1}$ The more detailed discussion of this integration can be found in the Appendix.
} 
At high energy colliders the distribution of particles inside the beams can be often considered as Gaussian. In this case $L(\varrho \mathbf{n})$ equals:

$$
L(\varrho \mathbf{n})=L \exp \left\{-\varrho^{2}\left(\frac{\cos ^{2} \varphi}{2 a_{x}^{2}}+\frac{\sin ^{2} \varphi}{2 a_{y}^{2}}\right)\right\}, \quad \mathbf{n}=(\cos \varphi, \sin \varphi, 0)
$$

where

$$
a_{x}^{2}=\sigma_{1 x}^{2}+\sigma_{2 x}^{2}, \quad a_{y}^{2}=\sigma_{1 y}^{2}+\sigma_{2 y}^{2} .
$$

This results in the following expression for $a$ :

$$
a=\sqrt{\frac{\pi}{2}} \frac{a_{x} a_{y}}{\sqrt{a_{y}^{2} \cos ^{2} \varphi+a_{x}^{2} \sin ^{2} \varphi}} .
$$

For the round (but not identical) beams with the root-mean-square radii

$$
\sigma_{1 x}=\sigma_{1 y}=\sigma_{1 \perp}, \quad \sigma_{2 x}=\sigma_{2 y}=\sigma_{2 \perp}
$$

we have

$$
a=\sqrt{\frac{\pi}{2}} \sqrt{\sigma_{1 \perp}^{2}+\sigma_{2 \perp}^{2}} .
$$

It is interesting to note that the quantity $a$ and the non-standard cross section are determined by the size of the largest beam. For the round and identical beams with $\sigma_{1 \perp}=\sigma_{2 \perp}=\sigma_{\perp}$ the result of (23) can be obtained.

\section{The cross section of the $\mu^{-} \mu^{+} \rightarrow e \bar{\nu}_{e} W^{+}$reaction}

Let us analyze a special example taking $X=W^{+}$. The cross section for the reaction $\nu_{\mu} \mu^{+} \rightarrow W^{+}$can be written in the form

$$
\sigma_{\nu \mu \rightarrow W}=12 \pi^{2} \frac{\Gamma(W \rightarrow \mu \nu)}{M} \delta\left(x s-M^{2}\right)
$$

where $\Gamma\left(W \rightarrow \mu \bar{\nu}_{\mu}\right)=0.22 \mathrm{GeV}$ is the partial $W$ decay width and $M=80.2 \mathrm{GeV}$ is the $W$ boson mass. Integration over the fraction of the neutrino energy $x$ in the eq. (27) becomes trivial and we obtain:

$$
\begin{aligned}
\sigma_{\text {non-stand }}\left(\mu^{-} \mu^{+}\right. & \left.\rightarrow e \bar{\nu}_{e} W^{+}\right)=\frac{\pi a}{2 c \tau} \sigma_{0} x_{0} f\left(x_{0}\right), \quad x_{0}=\frac{M^{2}}{s}, \\
\sigma_{0} & =\frac{12 \pi^{2}}{M^{2}} \frac{\Gamma(W \rightarrow \mu \nu)}{M}=20 \mathrm{nb} .
\end{aligned}
$$

For numerical estimates we take $a=\sqrt{\pi} \sigma_{\perp}$ (which corresponds to the case of the round identical Gaussian beams) with (see ref. [8])

$$
\sigma_{\perp}=10^{-3} \mathrm{~cm}
$$

This non-standard cross section reaches the maximum of 0.76 fb for $\sqrt{s}=93 \mathrm{GeV}$. For larger energies this cross section decreases as $s^{-3 / 2}$. It is interesting to note that the 
modest value of the non-standard cross section $0.76 \mathrm{fb}$ is the result of the product of the very small number of neutrinos

$$
N_{\nu}=\frac{\pi \sqrt{\pi}}{2} \frac{\sigma_{\perp}}{c \tau}=4.2 \cdot 10^{-8}
$$

and the huge value of the cross section for the $\nu_{\mu} \mu^{+} \rightarrow W^{+}$transition averaged over neutrino spectrum

$$
\left\langle\sigma_{\nu \mu \rightarrow W}\right\rangle=\sigma_{0} x_{0} f\left(x_{0}\right)=1.8 \cdot 10^{7} \mathrm{fb}, \quad x_{0}=0.74
$$

First, let us compare this non-standard piece with the standard contribution to the same cross section. We remind that by "standard" contribution we mean the cross section of the same reaction calculated by the standard rules excluding the region of the final phase space where $q^{2}>-m^{2}$. This contribution was calculated [9] with the help of the CompHEP package [10]. The comparison of both contributions is shown in the fig.3. It is seen that the non-standard contribution dominates up to the energies $\sqrt{s} \approx 105 \mathrm{GeV}$.

Second, we compare our non-standard cross section with the cross section for the single $W$ boson production in the reaction $\mu^{-} \mu^{+} \rightarrow \mu^{-} \bar{\nu}_{\mu} W^{+}$. The latter is completely standard process since it has no $t$-channel singularity. The reasonable estimate of its cross section can be quickly obtained with the help of the equivalent photon approximation. It gives the cross section $\approx 1 \mathrm{fb}$ at $\sqrt{s} \approx 95 \mathrm{GeV}$ (where it almost coincides with our non-standard cross section). At higher energies the process $\mu^{-} \mu^{+} \rightarrow \mu^{-} \bar{\nu}_{\mu} W^{+}$dominates as compared with the discussed process $\mu^{-} \mu^{+} \rightarrow e \bar{\nu}_{e} W^{+}$.

\section{Conclusions}

We have considered the processes with the $t$-channel singularity in the physical region. As the result of our study the following statement is proved:

Accounting for the finite beam sizes eliminates the singularity and makes it possible to obtain the finite result for the observable cross section.

In contrast to all previously known cases, the beam-size effect for the processes with the $t$-channel singularity in the physical region turns out to be linear proportional to the transverse size of the colliding beams.

The effect appears to be connected with the real decay of the unstable particle in the initial state followed by the scattering of its decay products on the opposite beam [6]. Evidently, the "interaction region" for such a scenario is only bounded if the finite sizes of the colliding beams are taken into account. In the traditional approach to the scattering process the initial states are the plane waves, which are not restricted to any finite volume. As a price, infinite cross section due to the $t$-channel singularity is obtained.

The results presented in this paper correspond to the situation when the parameter $a /(c \tau) \sim N_{\nu} \ll 1$ or when the unstable particle life time is much larger than the interaction time ( for muon colliders this approximation is clearly perfect). If this parameter increases, stationary approximation becomes wrong at some point. In this case the effects of the initial particle instability and the beam size effects should be considered simultaneously.

As a practical example, we consider the process of the single $W$ boson production at $\mu^{-} \mu^{+}$colliders. We demonstrate that the standard cross section for a single $W$ boson production and the non-standard one are of the same order of magnitude in the energy 
region slightly above threshold of this reaction. For higher energies the relative size of the non-standard contribution rapidly decreases.

We also note that precisely in the same way as above the processes like $\mu^{-} \mu^{+} \rightarrow e \bar{\nu}_{\mu} W^{+}$ and $\mu^{-} \mu^{+} \rightarrow \nu_{\mu} \bar{\nu}_{e} W^{+}$can be considered. In this processes the $t$-channel singularity will be due to the electron neutrino and electron propagators respectively. In virtue of the discussion given above these processes will correspond to the collision of the real electron neutrino or electron produced in the $\mu^{-}$decay with the opposite $\mu^{+}$beam.

\section{Acknowledgments}

K.M. is grateful to the Graduiertenkolleg "Teilchenphysik", Universität Mainz for support and to E. Sherman for a number of fruitful discussions. V.G.S. acknowledges support of the Sächsisches Staatsministerium für Wissenschaft und Kunst, of the Russian Fund of Fundamental Research and of INTAS 93-1180. We thank V.S. Fadin, I.F. Ginzburg and G.L. Kotkin for valuable discussions. We are grateful to E.E. Boos and A.E. Pukhov for providing us with the results of the CompHEP calculations.

\section{References}

[1] R.F. Peierls, Phys. Rev. Lett. 6 (1961), 641

[2] I.F. Ginzburg, Preprint DESY 95-168 (1995), hep-ph 9509314

[3] G.L. Kotkin, V.G. Serbo and A. Schiller, Int. Journ. Mod. Phys. A 7 (1992), 4707

[4] A.E. Blinov et al., Phys. Lett. B 113 (1982), 423

[5] K. Piotrzkowski, Preprint DESY 95-051 (1995)

[6] Such interpretation was pointed to us by G.L. Kotkin

[7] G.L. Kotkin, S.I. Polityko and V.G. Serbo, Yad. Fiz. 42 (1985), 692

[8] D.Cline, Nucl. Instrum. Methods 350 (1995), 24; R.B. Palmer, Beam Dynamics Newsletters 8 (1995)

[9] E.E. Boos and A.E. Pukhov, Private communication

[10] E.Boos et al, Preprint SNUTP-94-116, hep-ph/9503280

\section{Appendix: The model example}

In this appendix a simple example of the reaction $2 \rightarrow 2$ with the $t$-channel singularity in the physical region is considered in detail. The same notations as in the main text of the paper are used. We work within the frame of the scalar field theory with the interaction Lagrangian

$$
\mathcal{L}_{I}(x)=g A(x) B(x) C(x) .
$$

The fields $A, B$ and $C$ represent the particles with the masses $M, m$ and zero respectively. For definiteness we assume $M>m$. To avoid confusion, note also that the coupling $g$ 
has a dimension of mass. The particle $A$ is unstable due to the $A \rightarrow B+C$ process, its decay width is of the order

$$
\Gamma \sim \frac{g^{2}}{16 \pi M}
$$

Let us consider the scattering of the particles $A$ and $B$ :

$$
A\left(p_{1}\right)+B\left(p_{2}\right) \rightarrow B\left(p_{3}\right)+A\left(p_{4}\right) .
$$

The matrix element of this process corresponds to the Feynman diagrams with the $C$ particle exchange in the $t$ - and $s$-channels. For high energies, $s \gg M^{2}, m^{2}$, the dominant contribution comes from the diagram with the $C$-particle exchange in the $t$-channel:

$$
M=-\frac{g^{2}}{q^{2}+i \epsilon} .
$$

Simple kinematical relations provide the following limits for the square of the momentum transfer $q^{2}$ in the $t$-channel:

$$
-s \leq q^{2} \leq t_{0}=\frac{\left(M^{2}-m^{2}\right)^{2}}{s} .
$$

Hence, the value of $q^{2}$ goes through zero. As was already indicated in the paper, in this case the cross section can not be calculated by means of the standard methods. It is easy to see, that $q^{2}=0$ for the value of the transverse momentum

$$
\left|\mathbf{q}_{\perp}\right|=q_{\perp}^{0}=\sqrt{t_{0}}=\frac{M^{2}-m^{2}}{\sqrt{s}} .
$$

To model the real situation discussed above, the following conditions have to be fulfilled:

(i) The unstable particle life time must be much larger than the interaction time, i.e. (compare eqs. (3) and (22))

$$
M \Gamma \ll \frac{\sqrt{t_{0}}}{a} .
$$

(ii) The transverse beam size $a$ must be large in comparison with the typical inverse transverse momentum (51), i.e.

$$
a \gg \frac{1}{\sqrt{t_{0}}} .
$$

Taking into account (48) and introducing small parameter $\delta$ we express both of these constrains in the form

$$
\frac{g^{2}}{16 \pi t_{0}} \ll \delta=\frac{1}{a \sqrt{t_{0}}} \ll 1
$$

We now apply the same technique, as in the main text of the paper. We start from the complete description of the scattering process in terms of the colliding beams (see eqs. 29)(32)) and calculate the quantity $\sigma(\boldsymbol{\kappa})$. For this aim we expand $t$-channel propagators up to the terms of the order $\boldsymbol{\kappa}$, exactly as it has been done in the main text. Then the following formulae are valid:

$$
M_{f i}=-\frac{g^{2}}{t+i \epsilon}, \quad M_{f i^{\prime}}=-\frac{g^{2}}{t^{\prime}+i \epsilon}
$$


where the quantities $t$ and $t^{\prime}$ are given in (34)-(37). To calculate

$$
\frac{d \sigma(\boldsymbol{\kappa})}{d \varphi}=\int_{-s}^{t_{0}} \frac{M_{f i} M_{f i^{\prime}}^{*}}{32 \pi^{2} s^{2}} d q^{2}
$$

we have to calculate the integral

$$
J=\int_{-s}^{t_{0}} \frac{d q^{2}}{D\left(q^{2}\right)}, \quad D\left(q^{2}\right)=(t+i \epsilon)\left(t^{\prime}-i \epsilon\right)=\left(q^{2}-\boldsymbol{\kappa} \mathbf{q}_{\perp}+i \epsilon\right)\left(q^{2}+\boldsymbol{\kappa} \mathbf{q}_{\perp}-i \epsilon\right) .
$$

Here the quantity

$$
\boldsymbol{\kappa} \mathbf{q}_{\perp}=\boldsymbol{\kappa} \mathbf{n} \sqrt{t_{0}-q^{2}}
$$

depends both on $q^{2}$ and $\varphi$. To evaluate $J$ in the limit of the small momenta $\boldsymbol{\kappa}$, we rewrite it in the following way:

$$
J=\int_{C} \frac{d q^{2}}{D\left(q^{2}\right)}-\int_{-\infty}^{-s} \frac{d q^{2}}{D\left(q^{2}\right)}-\int_{t_{0}}^{\infty} \frac{d q^{2}}{D\left(q^{2}\right)}
$$

where the contour $C$ goes around the upper half plane $?$.

The integral over the contour $C$ can be evaluated by using Cauchy's theorem. The result is provided by the pole in the upper half plane

$$
\int_{C} \frac{d q^{2}}{D\left(q^{2}\right)}=\frac{2 \pi i}{R}
$$

where

$$
R=\frac{d D\left(q^{2}\right)}{d q^{2}} \quad \text { at } \quad q^{2}+\boldsymbol{\kappa} \mathbf{q}_{\perp}-i \epsilon=0 .
$$

Taking into account the terms of the order $\delta$ and neglecting the terms of the order $\delta^{2}$, we obtain

$$
R=2\left(-\kappa \mathbf{n} \sqrt{t_{0}}+i \epsilon\right) .
$$

As for the integrals both from $t_{0}$ to the $\infty$ and from the $-\infty$ to $-s$, we note that the dependence of the integrand on $\boldsymbol{\kappa}$ can be neglected and consequently

$$
\int_{-\infty}^{-s} \frac{d q^{2}}{D\left(q^{2}\right)}+\int_{t_{0}}^{\infty} \frac{d q^{2}}{D\left(q^{2}\right)} \approx \frac{1}{t_{0}}
$$

As a result, we obtain

$$
\frac{d \sigma(\boldsymbol{\kappa})}{d \varphi}=\frac{g^{4}}{32 \pi^{2} s^{2}}\left(\frac{\pi i}{-\boldsymbol{\kappa} \mathbf{n} \sqrt{t_{0}}+i \epsilon}-\frac{1}{t_{0}}\right) .
$$

Using representation (41) we get after trivial integration over $\boldsymbol{\kappa}$ and $\varrho$

$$
\frac{d \sigma(A B \rightarrow B A)}{d \varphi}=\frac{g^{4}}{32 \pi^{2} s^{2}}\left(\frac{\pi a}{\sqrt{t_{0}}}-\frac{1}{t_{0}}\right)
$$

where the quantity $a$ is defined in (42). Generally speaking, $a$ can depend on the angle $\varphi$ (see (44)). Note that the "beam-size" dependent part dominates in the cross section (63) due to the inequality (53).

\footnotetext{
${ }^{2}$ Note, that due to the eq.(56) the last integral in the eq.(57) also requires some definition. However for our purposes the dependence of this integral on $\boldsymbol{\kappa}$ is not important. Hence the last integral in the
} 
eq.(57) should be understood as the value of this integral for $\kappa=0$. 


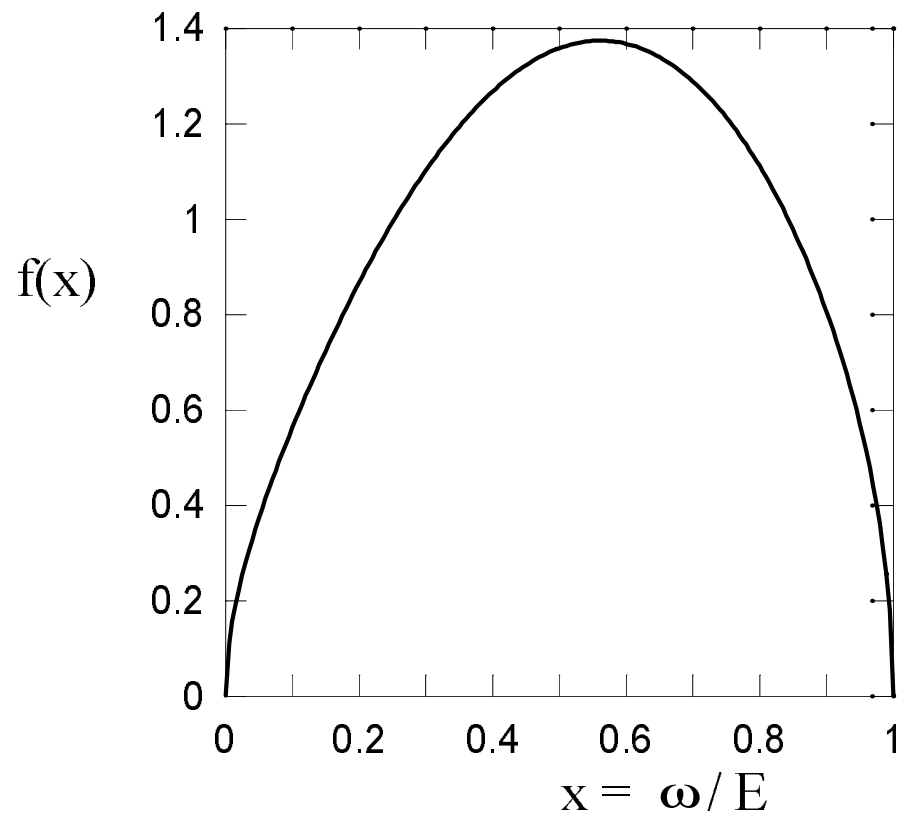

Figure 2: The normalized spectrum of neutrinos $f(x)=\left(1 / N_{\nu}\right)\left(d N_{\nu} / d x\right)$ vs. $x$ - the fraction of the muon energy carried by neutrino (see eq.(25)). 


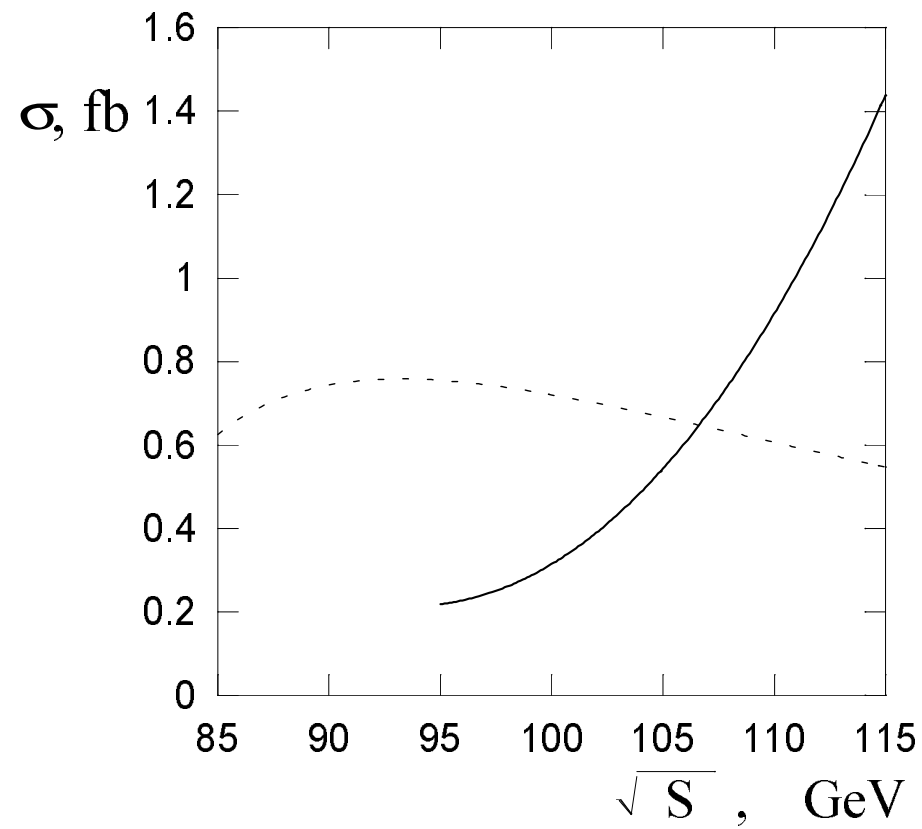

Figure 3: Standard (solid line) and non-standard (dashed line) contributions to the cross sections (fb) of the reaction $\mu^{-} \mu^{+} \rightarrow e \bar{\nu}_{e} W^{+}$in dependence on the total cms energy. The standard contribution is evaluated with the cut $-q^{2}>m^{2}$. 